\title{
Severity of Lower Urinary Tract Symptoms among Middle Aged and Elderly Nigerian Men: Impact on Quality of Life
}

\author{
Patrick Temi Adegun, ${ }^{1}$ Philip Babatunde Adebayo, ${ }^{2}$ and Peter Olufemi Areo ${ }^{1}$ \\ ${ }^{1}$ Department of Surgery, College of Medicine, Ekiti State University, Ekiti State 36001, Nigeria \\ ${ }^{2}$ Department of Medicine, Ladoke Akintola University of Technology, Ogbomoso, Oyo State, Nigeria \\ Correspondence should be addressed to Patrick Temi Adegun; patrickikelomo@yahoo.com
}

Received 31 March 2016; Accepted 31 May 2016

Academic Editor: Matthew Rutman

Copyright (c) 2016 Patrick Temi Adegun et al. This is an open access article distributed under the Creative Commons Attribution License, which permits unrestricted use, distribution, and reproduction in any medium, provided the original work is properly cited.

Objectives. To compare the severity of LUTS among middle aged and elderly Nigerian men and determine the influence of LUTS severity on QoL. Methods. This cross-sectional study was conducted among new patients presenting with LUTS attending Urology clinic between 2011 and 2015. Assessment of symptoms was based on IPSS and bother score completed by the eligible subjects on the same day of their clinic visits. Results. Four hundred patients were studied comprising 229 middle aged and 171 elderly men. Interquartile range (IQR) of IPSS scores for men $<65$ years and those $\geq 65$ years was $14.0(16.0)$ and $19(15.0)$, respectively $(p<0.001)$. Mild LUTS was significantly associated with best, good, and poor quality of life while moderate LUTS was associated with poor QoL. Severe LUTS was significantly associated with all the categories of QoL (Best-Worst). Among the cohort of subjects with poor QoL, elderly patients had a significantly higher median IPSS score $(p<0.05)$. Conclusions. There is no level of severity of LUTS in which patients' QoL is not impaired although mild symptomatology may be associated with better QoL and severe symptomatology with poor QoL. Careful attention to QoL may help identify patients who require early and prompt treatment irrespective of the IPSS.

\section{Introduction}

Olmsted County study, one of the largest longitudinal studies conducted in America, investigated age as one of many sociodemographic characteristics that may predict the incidence of LUTS/BPH. Also, age was reported to be one of the most reliable risk factors for the progression of LUTS/BPH. Its influence is greater than those of other sociodemographic characteristics [1].

Aging as a process has been discovered to be associated with the development of various, sometimes distressing, symptoms of different organ systems in the body including the genitourinary tract [2].

Besides, European and Korean EPIC studies have estimated that about 2/3rd of LUTS is present in the middle aged men whereas other researchers reported that the conditions leading to LUTS are among the most prevalent diseases of the elderly, with serious impairment of quality of life [QoL] [1,3-15].

However, as men age, there is an increasing prevalence of Lower Urinary Tract Symptoms (LUTS) but no clear difference in the impact of LUTS on the QoL between the middle aged and the elderly men [16].

Therefore, there is the need to differentiate between the effects of severity of LUTS on the quality of life of middle aged and the elderly men. Comparison of impact of LUTS in these men is important to improve the management of LUTS in men, some of whom are still in the working class and the bread winners for their families.

The objective of this study was to compare the severity of LUTS among middle aged and elderly Nigerian men attending the Urology clinic at Ekiti State University Teaching Hospital, South Western Nigeria. We also aimed to determine the influence of LUTS severity on the QoL of these men.

\section{Methods}

2.1. Settings. This was a comparative cross-sectional study carried out on all new patients who presented to the Urology clinic of Ekiti State University Teaching Hospital, Ado-Ekiti, South Western Nigerian from July 1, 2011, to June 30, 2015. 
2.2. Selection Criteria and Data Collection. The inclusion criteria were male sex; 40 years aged and above; voluntary participation; and understanding and signing the consent form. The exclusion criteria were previous open prostatectomy; acute diseases such as sepsis syndrome, cardiovascular events, and trauma; surgeries or hospitalizations during the preceding month; and uncompensated chronic diseases.

A questionnaire containing sociodemographics and concurrent medical conditions including history of alcohol ingestion and 8-item International Prostate Symptoms Score (IPSS) (English version was used because official national language in Nigeria is English) was completed by the eligible subjects on the same day of their clinic visits. The IPSS is a reliable and widely used instrument since 1991, and it is based on the answers to seven questions concerning urinary symptoms and one question concerning quality of life [17]. Each question concerning urinary symptoms allows the patient to choose one out of six answers indicating increasing severity of the particular symptom. The answers are assigned points from 0 to 5 . The total score ranges from 0 to 35 (asymptomatic to very symptomatic). The questions refer to the following urinary symptoms: (i) incomplete emptying, (ii) frequency, (iii) intermittency, (iv) urgency, (v) weak stream, (vi) straining, and (vii) nocturia. Question 8 measures patient's perceived quality of life (QoL) which comprises seven answers from 0 to 6 . The quality of life or level of satisfaction of LUTS of patients was represented by seven grades: "no problem" (0 point = very satisfied), "I'm all right" (1 point), "somewhat satisfied" ( 2 points), "half satisfied, half dissatisfied" (3 points), "somewhat dissatisfied" (4 points), "distressed" (5 points), and "I can't stand it" (6 points = very dissatisfied).

Self-administration of questionnaires was preferred, but face-to-face interviews were conducted whenever the participants presented with visual deficits, illiteracy, or semiilliteracy that would preclude them from proper completion of the questionnaire. Thirteen (13) participants had their questionnaire interviewer administered. Trained medical staff conducted the interviews in private rooms, which lasted an average of 30 minutes.

2.3. Ethical Issues. The study was conducted in accordance with Declaration of Helsinki (as revised in Edinburgh 2000). Written informed consent was obtained from all participants before participation in the study.

Ethical approval was obtained from Ethical Research Committee of the Ekiti State University Teaching Hospital, Ado-Ekiti, Nigeria.

2.4. Statistical Analysis. For statistical analysis, the subject's demographic and clinical variables were summarized and presented as frequencies and percentages for categorical variables while numerical data were summarized as means and standard deviation when normally distributed and median with interquartile range (IQR) when skewed. IPSS was categorized into mild (0-7), moderate (8-19) and severe symptoms (20-35) while quality of life (QoL) defined by bother score was categorized as best for BS $=0-1$; good for $\mathrm{BS}=2-3$; poor for $\mathrm{BS}=4-5$; and worst for $\mathrm{BS}=6$.
Patients demographic and clinical data were summarized and presented as frequencies and percentages while the chi-squared test was used to test differences between the middle aged ( $<65$ years) and elderly men ( $\geq 65$ years). Skewed continuous variables were summarized as median (interquartile range) while Mann-Whitney $U$ nonparametric test was used to test the differences in the median values between middle aged and elderly subjects. Chi-squared test was used to analyse the difference between quality of life categories (bother score) and severity of LUTS (defined by IPSS). To detect the significant group, the chi-squared test was followed by a multiple pairwise comparison test with adjustment of the $p$ values. All statistical analyses were performed using SPSS version 20.0 (SPSS, Chicago, Illinois). A $p$ value $<0.05$ was considered to be statistically significant while adjusted $p$ value of $<0.0042$ was considered significant for the post hoc pairwise comparison.

\section{Results}

Table 1 showed that while elderly men were either married or widowers or single or divorced men could be found among the middle aged. This finding was statistically significant. In addition, majority of the elderly men were retired. While majority of the middle aged cohort were overweight, normal weight and obese men were more common among the elderly. This was also significant. Among the comorbidities studied, systemic hypertension and diabetes mellitus were more prevalent in the elderly, while alcohol consumption was prevalent in the middle aged men. Table 2 showed that the severity of LUTS was significantly worse among subjects of 65 years and above $(p<0.05)$.

Table 3 showed the quality of life of patients with LUTS in relation to the severity of the symptoms where the IPSS score was categorized. There was a statistically significant relationship between the severity of LUTS and the QoL of the subjects.

Table 4 shows the results of post hoc multiple pairwise comparisons of the categories of the contingency Table 3 after the chi-squared test has rejected the null hypothesis of equality of proportions of subjects across the cells. The table showed the pairwise proportions among the multiple cross-classifications that led to the statistical significance observed in Table 3 after adjusting the $p$ value to 0.0042 . Mild symptomatic was significantly associated with best, good, and poor quality of life while moderate symptomatic was associated with poor QoL. Severe symptomatic was significantly associated with the entire categories of QoL (Best-Worst).

Figure 1 showed the IPSS scores among the subjects with different perceived QoL according to the age group. Among the cohort with best quality of life, the elderly patients had a statistically significant median IPSS score $(p>0.05)$. No statistical difference exists between the median IPSS scores of the cohort who had good QoL $(p>0.05)$. Among the cohort of subjects with poor QoL, elderly patients had a statistically significantly higher median IPSS score $(p>0.05)$.

\section{Discussion}

We sought to compare the severity of symptoms on the QoL of middle aged and elderly Nigerian men. This result showed 
TABLE 1: Demographic characteristics of the study population.

\begin{tabular}{|c|c|c|c|c|}
\hline Variables & Age $<65$ years; $229(\%)$ & Age $\geq 65$ years; $171(\%)$ & Test statistics & $p$ value \\
\hline Marital status & & & & $<0.001$ \\
\hline Single & $23(10)$ & $0(0)$ & 35.36 & \\
\hline Married & $193(84.3)$ & $166(97.1)$ & & \\
\hline Divorced & $13(5.7)$ & $0(0)$ & & \\
\hline Widower & $0(0)$ & $5(2.9)$ & & \\
\hline Occupation & & & 77.62 & $<0.001$ \\
\hline Public servant & $106(46.3)$ & $19(11.1)$ & & \\
\hline Business & $31(13.5)$ & $45(26.3)$ & & \\
\hline Retired & $39(17.0)$ & $79(46.2)$ & & \\
\hline Others & $53(23.1)$ & $28(16.4)$ & & \\
\hline \multicolumn{5}{|l|}{ BMI } \\
\hline Underweight & $0(0)$ & $1(0.6)$ & 11.21 & 0.011 \\
\hline Normal & $67(29.3)$ & $71(41.5)$ & & \\
\hline Overweight & $126(55.0)$ & $67(39.2)$ & & \\
\hline Obese & $36(15.7)$ & $32(18.7)$ & & \\
\hline \multicolumn{5}{|l|}{ Comorbidities } \\
\hline Hypertension & $120(52.4)$ & $115(67.3)$ & 8.91 & 0.003 \\
\hline Diabetes mellitus & $18(7.9)$ & $20(11.7)$ & 1.67 & 0.196 \\
\hline Alcohol & $91(39.7)$ & $36(21.1)$ & 11.05 & 0.001 \\
\hline \multicolumn{5}{|l|}{ Ultrasound findings } \\
\hline Normal size & $52(22.7)$ & $2(1.2)$ & 11.95 & 0.003 \\
\hline Enlarged and benign & $161(70.3)$ & $160(93.6)$ & & \\
\hline Suspected cancer & $16(7.0)$ & $9(5.3)$ & & \\
\hline DRE & & & 18.50 & $<0.001$ \\
\hline Normal sized prostate & $35(15.3)$ & $9(5.3)$ & & \\
\hline Enlarged and benign & $178(77.7)$ & $161(94.2)$ & & \\
\hline Suspicious lesion & $16(7.0)$ & $1(0.6)$ & & \\
\hline Diagnosis & & & 5.46 & 0.141 \\
\hline $\mathrm{BPH}$ & $181(79.0)$ & $141(82.5)$ & & \\
\hline $\mathrm{CaP}$ & $23(10.0)$ & $22(12.9)$ & & \\
\hline Urethral stricture & $6(2.6)$ & $2(1.2)$ & & \\
\hline Others for example OAB & $19(8.3)$ & $6(3.5)$ & & \\
\hline
\end{tabular}

$\mathrm{CaP}=$ cancer of the prostate; $\mathrm{OAB}=$ overactive bladder.

TABLE 2: IPSS, voiding, and storage subscores among the age group.

\begin{tabular}{lccc}
\hline Variables & $\begin{array}{c}\text { Age }<65 \text { years } \\
\text { Median (IQR) }\end{array}$ & $\begin{array}{c}\text { Age } \geq 65 \text { years } \\
\text { Median (IQR) }\end{array}$ & Mann-Whitney $U$ test \\
\hline IPSS & $14.0(16.0)$ & $19.0(15.0)$ & 15435.0 \\
Voiding symptoms & $7.0(12.0)$ & $9.0(10.0)$ & 16041.0 \\
Storage symptoms & $7.0(7.0)$ & $9.0(5.0)$ & 15804.5 \\
\hline
\end{tabular}

TABLE 3: Showing the quality of life of the subjects in relation to the severity of LUTS symptoms.

\begin{tabular}{|c|c|c|c|c|c|c|}
\hline \multirow[b]{2}{*}{ IPSS severity } & \multicolumn{4}{|c|}{ Bother score } & \multirow[b]{2}{*}{ Chi-squared test } & \multirow[b]{2}{*}{$p$ value } \\
\hline & $\begin{array}{c}\text { Best QoL } \\
n(\%)\end{array}$ & $\begin{array}{c}\text { Good QoL } \\
n(\%)\end{array}$ & $\begin{array}{c}\text { Poor QoL } \\
n(\%)\end{array}$ & $\begin{array}{c}\text { Worst QoL } \\
n(\%)\end{array}$ & & \\
\hline Mildly symptomatic & $26(68.4)$ & $42(55.3)$ & $12(4.3)$ & $0(0)$ & & \\
\hline Moderately symptomatic & $10(26.3)$ & $21(27.6)$ & $136(49.3)$ & $0(0)$ & 175.75 & $<0.001$ \\
\hline Severely symptomatic & $2(5.3)$ & $13(17.1)$ & $128(46.4)$ & $10(100)$ & & \\
\hline Total & $36(100)$ & $76(100)$ & $276(100)$ & $10(100)$ & & \\
\hline
\end{tabular}


TABLE 4: Pairwise comparison of IPSS severity and quality of life of patients with LUTS.

\begin{tabular}{lcc}
\hline Pair compared & $\begin{array}{c}\text { Chi- } \\
\text { squared } \\
\text { test }\end{array}$ & p value \\
\hline Mildly symptomatic versus best QoL & 60.84 & $<0.001^{*}$ \\
Mildly symptomatic versus good QoL & 72.25 & $<0.001^{*}$ \\
Mildly symptomatic versus poor QoL & 136.89 & $<0.001^{*}$ \\
Mildly symptomatic versus worst QoL & 2.56 & 0.109 \\
Moderately symptomatic versus best QoL & 4.00 & 0.046 \\
Moderately symptomatic versus good & 7.84 & 0.005 \\
QoL & & \\
Moderately symptomatic versus poor & 21.16 & $<0.001^{*}$ \\
QoL & & \\
Moderately symptomatic versus worst & 7.29 & 0.007 \\
QoL & & \\
Severely symptomatic versus best QoL & 19.36 & $<0.001^{*}$ \\
Severely symptomatic versus good QoL & 17.64 & $<0.001^{*}$ \\
Severely symptomatic versus poor QoL & 25.00 & $<0.001^{*}$ \\
Severely symptomatic versus worst QoL & 16.81 & $<0.001^{*}$ \\
\hline
\end{tabular}

Adjusted $p$ value $=0.0042$.

${ }^{*}$ statistically significant.

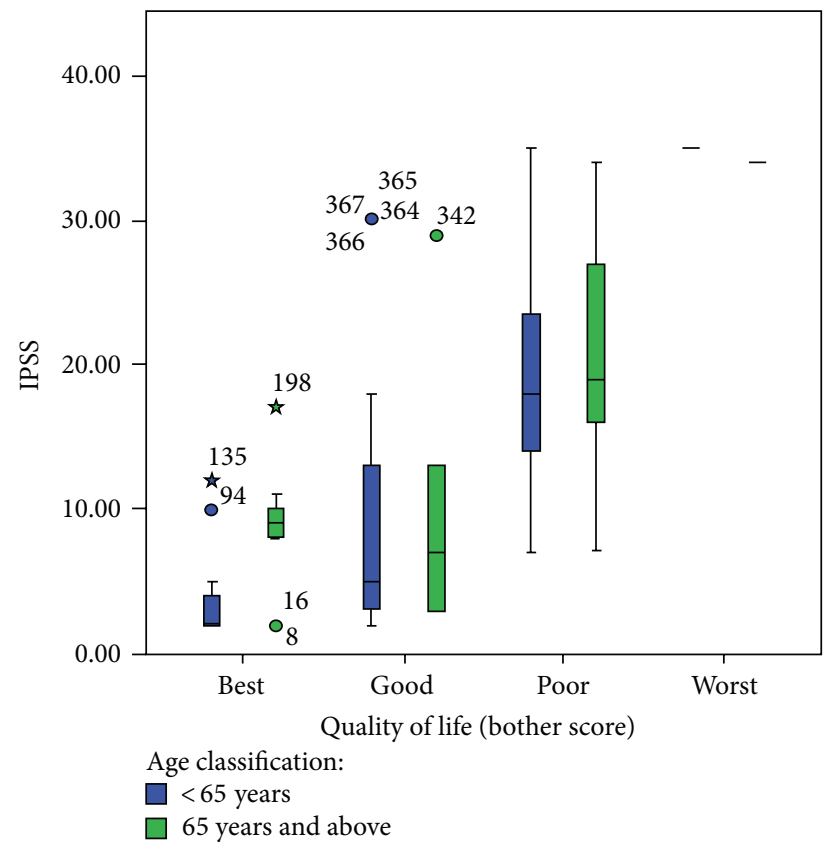

FIGURE 1: Showing quality of life versus age in years.

that QoL was affected by any category of symptoms (mild to severe) across the age. It was demonstrated in this study that even mild symptomatology of IPSS could be associated with poor quality of life whereas some severe symptomatology was associated with good QoL $(p<0.05)$. Therefore patients' health seeking behaviour might have been influenced by their QoL rather than the severity of the IPSS score. This finding is in agreement with the report of Finkelstein et al. that discovered that an individual's perceived impact on healthrelated quality of life might be a determinant for patients to seek medical advice [18].

In addition, the study also showed that severity of IPSS increases with age $(p<0.001)$. This is similar to the findings of Engström et al. that reported that severity of symptoms increases with age [19].

More importantly, among the cohort with best quality of life, the elderly patients had a statistically significant higher median IPSS score $(p<0.05)$. This might be due to high prevalence of hypertension in the elderly which could increase IPSS coupled with higher prevalence of alcohol consumption in the middle aged which might cause lower IPSS in them. However, a longitudinal study on the volume of alcohol consumption in this environment is necessary to corroborate this assertion. This is in line with Suh et al., $\mathrm{Lu}$, and Mo that reported a similar protective effect of lightmoderate alcohol consumption on IPSS [20,21].

Furthermore, this study showed that elderly men had higher frequency of poor QoL $(p<0.001)$. This is similar to the findings of Welch et al. that men with moderate and severe LUTS identified in a large US cohort had a poorer health status in several important quality of life dimensions [14]. It is important that these elderly men are adequately assessed for appropriate therapy. Surgical treatments have been strongly favoured for moderate to severe symptomatic patients and watchful waiting or conservative measures indicated for patients with mild complaints [22-24].

Recognition of significant deterioration of QoL among mild, moderate, and severe LUTS patients is an evidence for the need of treatment (as opposed to watchful and conservative approaches) and justification for early treatment irrespective of IPSS score. This is in consonance with Finkelstein et al. that noted that the IPSS quality of life question had a larger effect size than all the other measures suggesting that this single-item measure may have high sensitivity to differentiate subgroups [18].

\section{Conclusion}

From the foregoing, it might be better to use the QoL as determinant of the choice of treatment rather than the IPSS scores alone for prompt treatment of LUTS and improved clinical outcome.

\section{Limitation of the Study}

However, criticisms have been made regarding the poor standardization of QoL scales and the frequent inappropriate use of the term QoL. The use of a one-item scale to assess general QoL (the IPSS-QoL question, called "bother score") and the misinterpretation of QoL as synonymous to symptom-control or perceived general health or functional status are the most frequent reasons for such criticisms. Besides, because this study was hospital-based, it may not be a true random sample of men with LUTS in the general population. It is difficult to eliminate selection bias.

Furthermore, we did not evaluate for depressive symptomatology and other psychosocial contributors to QoL 
perception. However, the measure of QoL in this study is a disease specific instrument with psychometric properties well fitted to measure QoL as it relates to urinary symptoms.

\section{Competing Interests}

The authors have no conflict of interests.

\section{References}

[1] J. T. Wei, E. Calhoun, and S. J. Jacobsen, "Urologic diseases in America project: benign prostatic hyperplasia," Journal of Urology, vol. 179, no. 5, pp. S75-S80, 2008.

[2] E. Plas and L. K. Daha, "Hormonal replacement therapy for the aging bladder," in The Aging Bladder Day, E. Plas, Ed., pp. 137147, Springer, Wien, Austria, 2004.

[3] D. E. Irwin, I. Milsom, S. Hunskaar et al., "Population-based survey of urinary incontinence, overactive bladder, and other lower urinary tract symptoms in five countries: results of the EPIC study," European Urology, vol. 50, no. 6, pp. 1306-1315, 2006.

[4] Y.-S. Lee, K.-S. Lee, J. H. Jung et al., "Prevalence of overactive bladder, urinary incontinence, and lower urinary tract symptoms: results of Korean EPIC study," World Journal of Urology, vol. 29, no. 2, pp. 185-190, 2011.

[5] J. M. Fitzpatrick, "The natural history of benign prostatic hyperplasia," BJU International, vol. 97, no. 2, pp. 3-6, 2006.

[6] A. L. Burnett and A. J. Wein, "Benign prostatic hyperplasia in primary care: what you need to know," Journal of Urology, vol. 175, no. 3, pp. S19-S24, 2006.

[7] J. J. Medina, R. O. Parra, and R. G. Moore, "Benign prostatic hyperplasia (the aging prostate)," Medical Clinics of North America, vol. 83, no. 5, pp. 1213-1229, 1999.

[8] C. J. Girman, S. J. Jacobsen, T. Tsukamoto et al., "Health-related quality of life associated with lower urinary tract symptoms in four countries," Urology, vol. 51, no. 3, pp. 428-436, 1998.

[9] P. Trueman, S. C. Hood, U. S. L. Nayak, and M. F. Mrazek, "Prevalence of lower urinary tract symptoms and self-reported diagnosed 'benign prostatic hyperplasia', and their effect on quality of life in a community-based survey of men in the UK," BJU International, vol. 83, no. 4, pp. 410-415, 1999.

[10] A. Bertaccini, F. Vassallo, F. Martino et al., "Symptoms, bothersomeness and quality of life in patients with LUTS suggestive of BPH," European Urology, vol. 40, no. 1, pp. 13-18, 2001.

[11] A. S. Salinas-Sánchez, I. Hernández-Millán, J. G. LorenzoRomero, M. Segura-Martín, C. Fernández-Olano, and J. A. Virseda-Rodriguez, "Quality of life of patients on the waiting list for benign prostatic hyperplasia surgery," Quality of Life Research, vol. 10, no. 6, pp. 543-553, 2001.

[12] K. F. Quek, C. S. Loh, W. Y. Low, and A. H. Razack, "Quality of life assessment before and after transurethral resection of the prostate in patients with lower urinary tract symptoms," World Journal of Urology, vol. 19, no. 5, pp. 358-364, 2001.

[13] K. Yoshimura, Y. Arai, K. Ichioka, N. Terada, Y. Matsuta, and K. Okubo, "Symptom-specific quality of life in patients with benign prostatic hyperplasia," International Journal of Urology, vol. 9, no. 9, pp. 485-490, 2002.

[14] G. Welch, K. Weinger, and M. J. Barry, "Quality-of-life impact of lower urinary tract symptom severity: results from the Health Professionals Follow-up Study," Urology, vol. 59, no. 2, pp. 245250, 2002.
[15] A. F. Bobé, A. M. E. Buil, B. A. I. Allué, G. A. Morro, E. M. Maxenchs, and B. M. Gens, "Benign prostate hyperplasia. Need to evaluate quality of life in the therapeutic process," Atencion Primaria, vol. 38, no. 7, pp. 387-391, 2006.

[16] N. M. Resnick, "Urinary incontinence," The Lancet, vol. 346, no. 8967, pp. 94-99, 1995.

[17] M. J. Barry, F. J. Fowler Jr., M. P. O’Leary, R. C. Bruskewitz, H. L. Holtgrewe, and W. K. Mebust, "Measuring disease-specific health status in men with benign prostatic hyperplasia. Measurement Committee of the American Urological Association," Medical Care, vol. 33, no. 4, Article ID AS145, 1995.

[18] M. M. Finkelstein, J. Skelly, J. Kaczorowski, and G. Swanson, "Incontinence quality of life instrument in a survey of primary care physicians," Journal of Family Practice, vol. 51, article 952, 2002.

[19] G. Engström, L. Henningsohn, G. Steineck, and J. Leppert, "Self-assessed health, sadness and happiness in relation to the total burden of symptoms from the lower urinary tract," BJU International, vol. 95, no. 6, pp. 810-815, 2005.

[20] B. Suh, D. W. Shin, S.-S. Hwang et al., "Alcohol is longitudinally associated with lower urinary tract symptoms partially via highdensity lipoprotein," Alcoholism: Clinical and Experimental Research, vol. 38, no. 11, pp. 2878-2883, 2014.

[21] Z. Lu and Z. Mo, "Association of alcohol consumption with lower urinary tract symptoms in a Chinese male population survey," Translational Andrology and Urology, vol. 3, supplement 1, Article ID AB106, 2014.

[22] A. L. Burnett and A. J. Wein, "Benign prostatic hyperplasia in primary care: what you need to know," The Journal of Urology, vol. 175, no. 3, pp. S19-S24, 2006.

[23] M. J. Barry, A. G. Mulley Jr., F. J. Fowler, and J. W. Wennberg, "Watchful waiting vs immediate transurethral resection for symptomatic prostatism. The importance of patient's preferences," The Journal of the American Medical Association, vol. 259, no. 20, pp. 3010-3017, 1988.

[24] M. Oelke, A. Bachmann, A. Descazeaud et al., "EAU guidelines on male lower urinary tract symptoms (LUTS), including benign prostatic obstruction (BPO)," in Proceedings of the Male Lower Urinary Tract, pp. 123-144, 2012. 


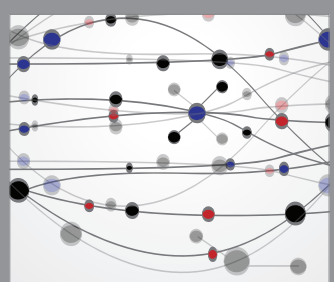

The Scientific World Journal
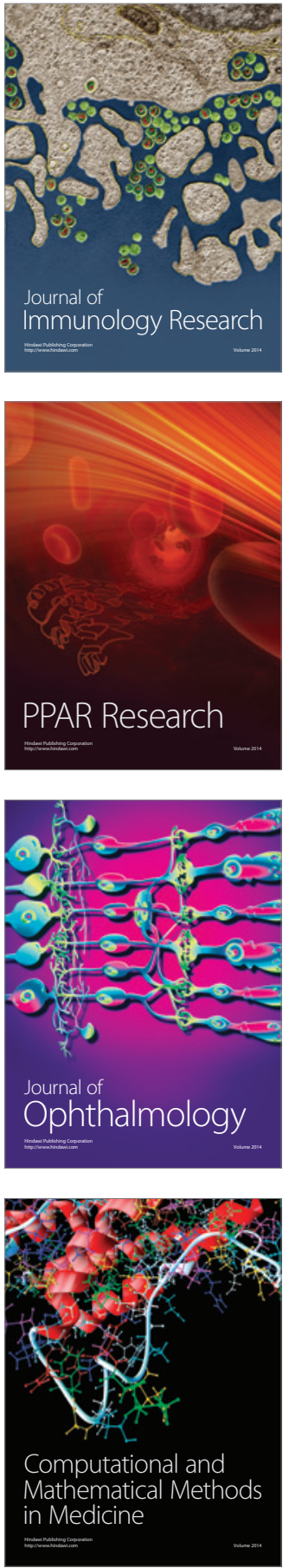

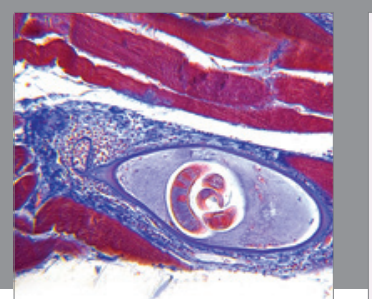

Gastroenterology Research and Practice

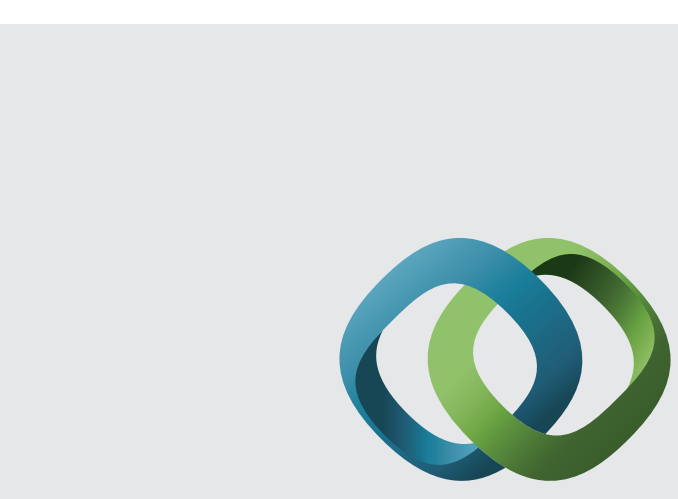

\section{Hindawi}

Submit your manuscripts at

http://www.hindawi.com
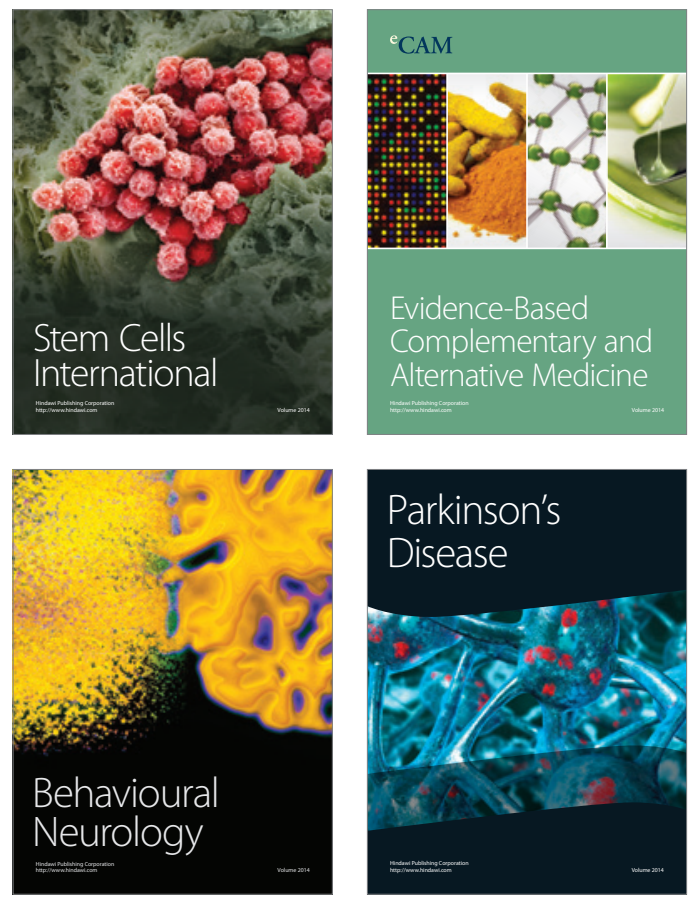
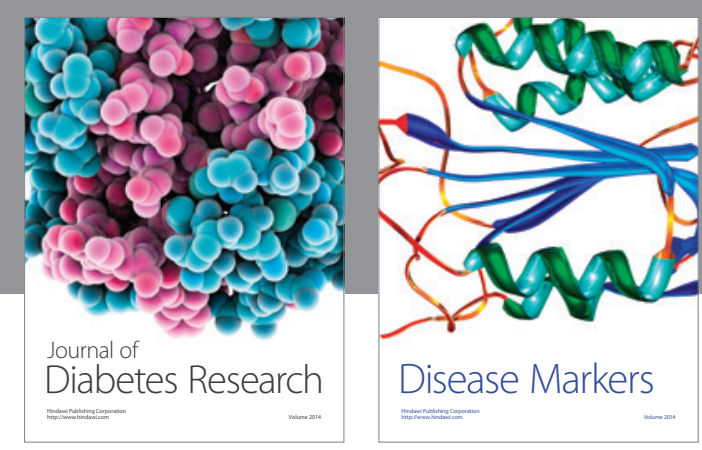

Disease Markers
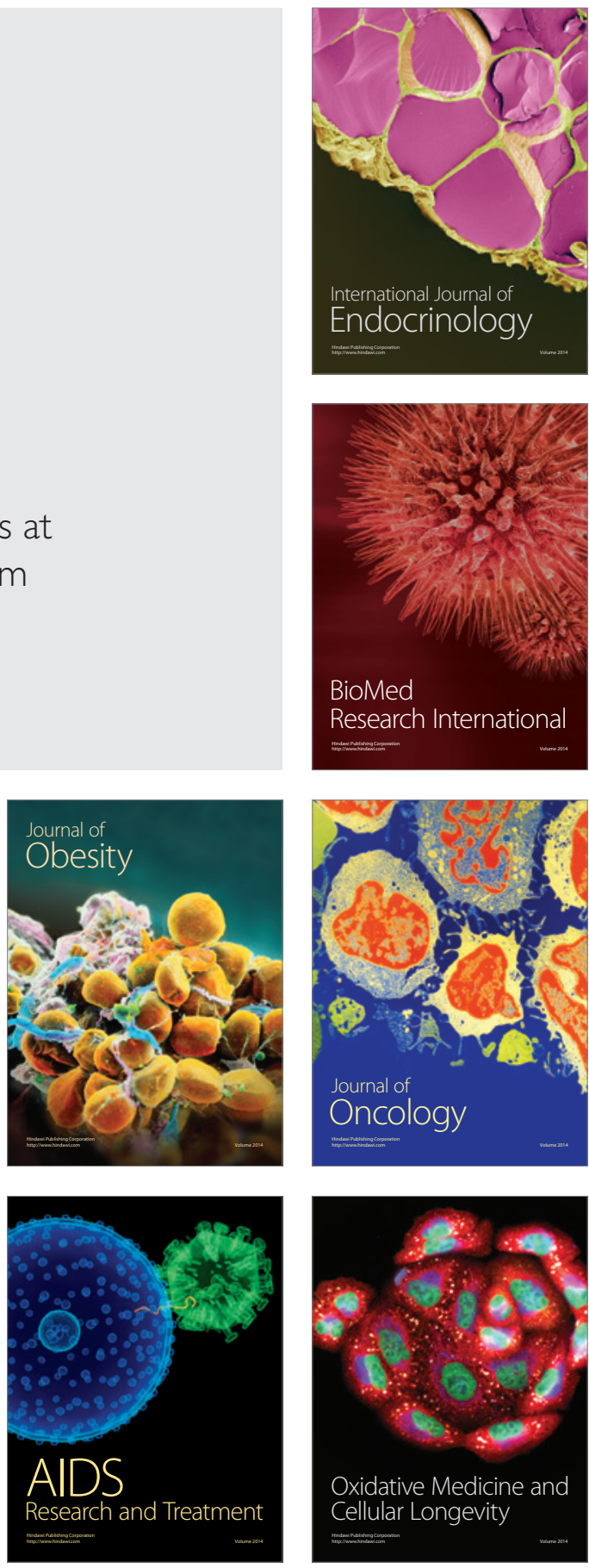